Supporting Information

\title{
Amorphous Nano-Hole Patterns Formed by Spinodal Decomposition of Nickel Superalloys for Solar-Selective Absorbers
}

Makoto Shimizu, ${ }^{a}{ }^{*}$ Zhen Liu, ${ }^{a}$ Hiroo Yugami ${ }^{a}$

${ }^{a}$ Graduate School of Engineering, Tohoku University, Aoba 6-6-01 Aramaki, Aoba-ku,

Sendai, 980-8579, Japan

*Corresponding author

E-mail: makoto.shimizu.a3@tohoku.ac.jp 


\section{Absorptance spectrum of bulk CMSX-4}

The optical constants of CMSX-4 was calculated from the Drude-model. In the calculation, the plasma frequency is assumed to be similar to that of nickel, the dominant element in CMSX-4, which is $1.43 \times 10^{16} \mathrm{rad} \mathrm{s}^{-1}$. The relaxation time $\tau$ is obtained by extrapolation of the calculated value for $\lambda<2.1 \mu \mathrm{m}$. Even with these assumptions, the measured absorptance shows good agreement with the simulated one.

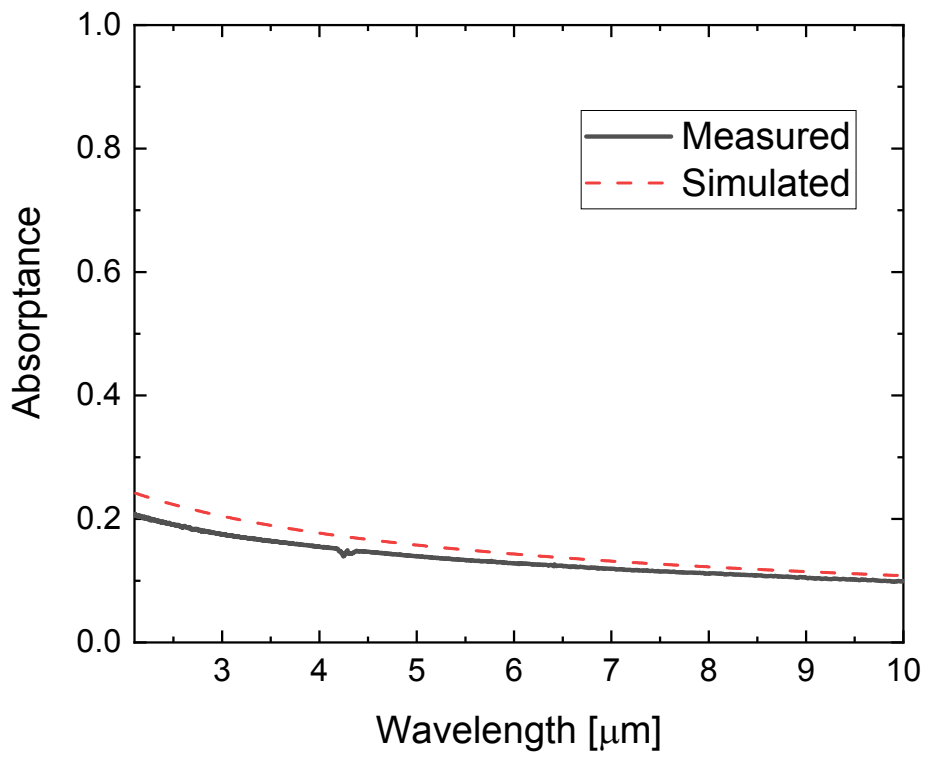

Figure S1. Absorptance spectra of the measured (black solid) and simulated (red dashed) bulk CMSX-4. 